\title{
Phase Fronts and Synchronization Patterns in Forced Oscillatory Systems
}

\author{
EHUD MERON* \\ The Jacob Blaustein Institute for Desert Research and the Physics Department, \\ Ben-Gurion University, Sede Boker Campus 84990, Israel
}

(Received 15 April 1999)

\begin{abstract}
This is a review of recent studies of extended oscillatory systems that are subjected to periodic temporal forcing. The periodic forcing breaks the continuous time translation symmetry and leaves a discrete set of stable uniform phase states. The multiplicity of phase states allows for front structures that shift the oscillation phase by $\pi / n$ where $n=1,2, \ldots$, hereafter $\pi / n$ fronts. The main concern here is with front instabilities and their implications on pattern formation. Most theoretical studies have focused on the $2: 1$ resonance where the system oscillates at half the driving frequency. All front solutions in this case are $\pi$-fronts. At high forcing strengths only stationary fronts exist. Upon decreasing the forcing strength the stationary fronts lose stability to pairs of counter-propagating fronts. The coexistence of counter-propagating fronts allows for traveling domains and spiral waves. In the $4: 1$ resonance stationary $\pi$-fronts coexist with $\pi / 2$-fronts. At high forcing strengths the stationary $\pi$-fronts are stable and standing two-phase waves, consisting of successive oscillatory domains whose phases differ by $\pi$, prevail. Upon decreasing the forcing strength the stationary $\pi$-fronts lose stability and decompose into pairs of propagating $\pi / 2$-fronts. The instability designates a transition from standing two-phase waves to traveling four-phase waves. Analogous decomposition instabilities have been found numerically in higher $2 n: 1$ resonances. The available theory is used to account for a few experimental observations made on the photosensitive Belousov-Zhabotinsky reaction subjected to periodic illumination. Observations not accounted for by the theory are pointed out.
\end{abstract}

Keywords: Forced oscillations, Resonances, Phase fronts, Instabilities, Patterns

\section{INTRODUCTION}

A conspicuous property of systems driven far from equilibrium is the possible appearance of persistent oscillations [1-5]. The onset of oscillations has extensively been studied in the context of chemical reactions. In spatially extended reactions it often involves spatial phase variations that lead to traveling wave phenomena. Biological rhythms provide another manifestation of persistent oscillatory dynamics. They occur in unicellular and multicellular organisms and cover a wide range of

\footnotetext{
*E-mail: ehud@bgumail.bgu.ac.il.
} 
periodicities, tens of milliseconds to years. The oscillating systems encountered in nature are not isolated and quite often the interaction with the environment takes the form of a periodic forcing in time. One example is the entrainment of cardiac cells in the atrioventricular node to signals generated at the sinoatrial node. Circadian rhythms entrained by the $24 \mathrm{~h}$ day-night periodicity provide another example.

Most theoretical studies of periodically forced oscillatory systems have focused on frequency locking phenomena and the onset of chaos in single oscillator models (or circle maps) [4,6-10]. Frequency locking refers to the property of a forced system to oscillate at a frequency $\omega$ which is a rational fraction of the forcing frequency $\omega_{\mathrm{f}}$ in some range of the latter. These ranges of resonant behavior get wider as the forcing strength is increased, and are commonly refer to as Arnold tongues. The fractional frequencies a forced system can realize follow the Farey rule: between the tongues $\omega_{\mathrm{f}}: \omega=n: m$ and $\omega_{\mathrm{f}}: \omega=k: l$ there exists the $(n+k):(m+l)$ tongue.

Another property of forced systems is the coexistence of multiple stable phase states, corresponding to uniform oscillations with different fixed phases. The multiplicity of phase states becomes particularly significant in coupled oscillator arrays or in oscillatory media, for different oscillator groups or spatial domains may oscillate with different phases, forming spatial patterns [11]. In the 2:1 tongue there are two stable phase states whose phases differ by $\pi$. They allow for two-phase patterns involving alternating domains of the two phase states. The boundaries between these alternating domains, hereafter $\pi$-fronts, have been studied recently by Coullet et al. [12]. They have found a front bifurcation reminiscent of the IsingBloch transition in ferromagnets with weak anisotropy. The bifurcation, now referred to as the nonequilibrium Ising-Bloch (NIB) bifurcation, renders a stationary (Ising) front unstable as the forcing strength is decreased, and gives rise to a pair of counter propagating (Bloch) fronts (see Fig. 3). The bifurcation designates a transition from standing two-phase patterns to traveling two-phase patterns [13].

In the $3: 1$ tongue there are three stable phase states giving rise to traveling three-phase patterns [14]. The boundaries between any pair of phase states form $2 \pi / 3$-fronts (shift the phase of oscillation by $2 \pi / 3$ ). The four stable phases in the $4: 1$ tongue allow for either two-phase or four-phase patterns depending on the forcing strength. At strong forcing stationary $\pi$-fronts are stable and standing two-phase patterns prevail. As the forcing strength is decreased stationary $\pi$-fronts lose stability and decompose into pairs of propagating $\pi / 2$ fronts (see Fig. 8). The $\pi$-front instability designates a transition from standing two-phase patterns to traveling four-phase patterns [15]. Recent experiments on the photosensitive Belousov-Zhabotinsky reaction subjected to periodic illumination demonstrate the existence of two and three-phase patterns [19]. Some of the observed patterns are shown in Fig. 1 .

In this paper I will review the mathematical analysis of the front instabilities described above, discuss the implications they bear on pattern formation, and use them to interpret some of the experimental observations. The mathematical analyses to be reviewed rely on continuum models of oscillatory systems. Such models apply not only to continuous media, such as chemical reactions, but also to discrete systems like coupled oscillator arrays when the coupling is strong enough. We will assume an instability of a uniform stationary state to uniform oscillations. Near the instability (the Hopf bifurcation) the system's dynamics is govern by a universal equation for the envelope (or amplitude) of the oscillations. We begin with a discussion of this equation.

\section{ENVELOPE EQUATION APPROACH}

Let $\mathbf{C}(x, t)$ represent the set of dynamical variables of a given system, and let $\mathbf{C}=\mathbf{C}_{\mathbf{0}}$ be a stationary uniform state of the system. We assume that the state $\mathbf{C}_{\mathbf{0}}$ loses stability, as a control parameter $R$ 


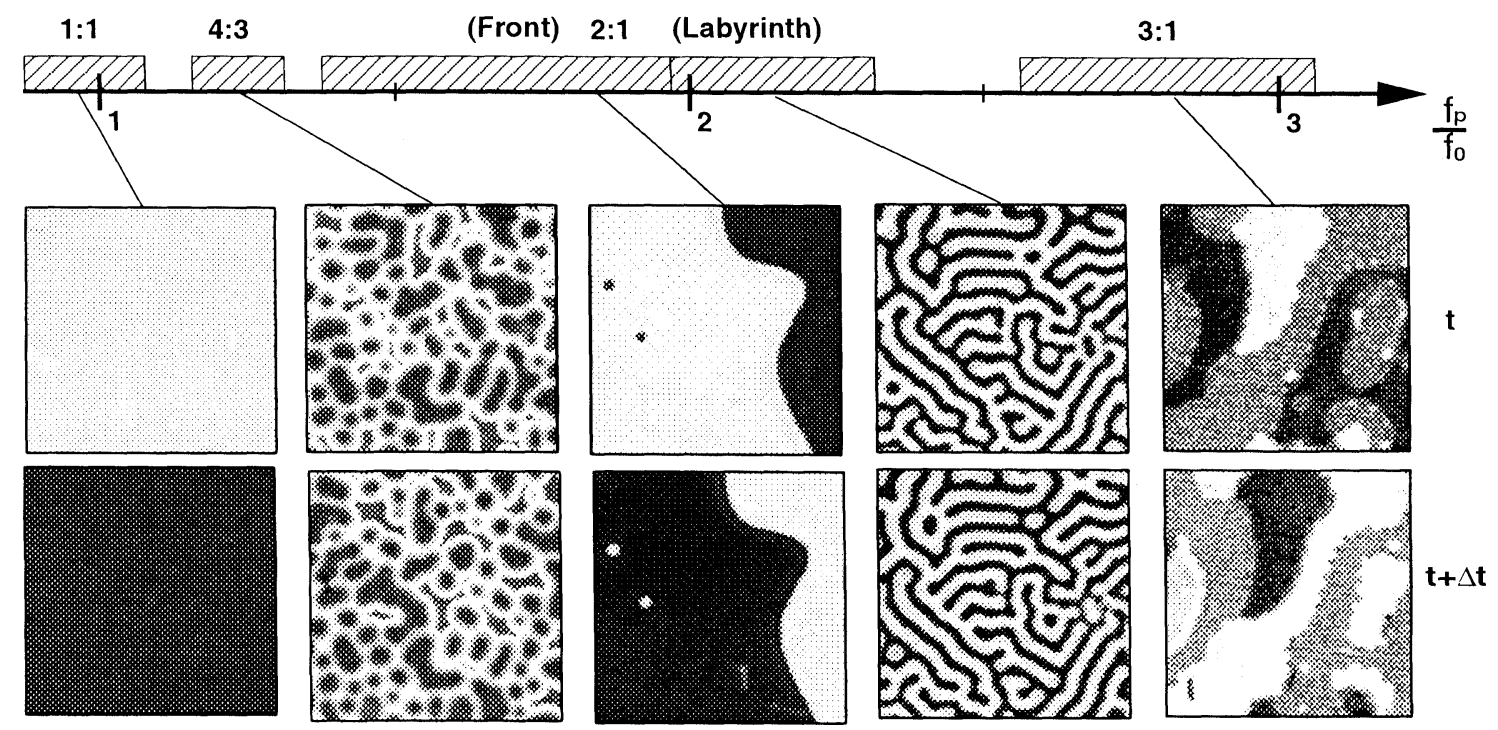

FIGURE 1 Experimental phase diagram for the ruthenium catalyzed Belousov-Zhabotinsky reaction periodically forced with pulses of spatially uniform light from a video projector. The diagram shows frequency locked regimes observed as a function of the forcing frequency, $f_{\mathrm{p}}\left(\omega_{\mathrm{f}}\right.$ in the text). Patterns are shown in pairs, one above the other, at times separated by $\Delta t=1 / f_{\mathrm{p}}$ except for the $1: 1$ resonance where $\Delta t=1 / 2 f_{\mathrm{p}}$. Striped boxes on the horizontal axis mark forcing frequency ranges with the same frequency-locking ratio. Reprinted by permission from Nature (Petrov et al., 388, 655-657) copyright (1997) Macmillan Magazines Ltd.

exceeds a critical value $R_{c}$, to uniform oscillations at frequency $\omega_{0}$ (Hopf bifurcation at zero wavenumber). Beyond the bifurcation and close to $R_{c}$ the set $\mathbf{C}(x, t)$ can be represented as an asymptotic expansion in powers of $\lambda=\sqrt{\left(R-R_{c}\right) / R_{c}} \ll 1$ :

$$
\mathbf{C}(x, t)=\mathbf{C}_{\mathbf{0}}+\lambda \mathbf{C}_{\mathbf{1}}+\lambda^{2} \mathbf{C}_{\mathbf{2}}+\lambda^{3} \mathbf{C}_{\mathbf{3}}+\cdots,
$$

where

$$
\mathbf{C}_{\mathbf{1}}=\mathbf{C}_{\mathbf{1 0}} A(x, t) \exp i \omega_{0} t+\text { c.c. }
$$

Here $\mathbf{C}_{\mathbf{1 0}}$ is a set of constants and "c.c." stands for the complex conjugate. The amplitude $A(x, t):=$ $\tilde{A}(X, T)$, where $X=\sqrt{\lambda} x$ and $T=\lambda t$, depends weakly on space and time. The slow temporal variations of $A$ stem from the small growth rates of perturbations near the bifurcation point, and the weak spatial dependence is a consequence of the narrow band of growing wavenumbers.

Assume now that the system is periodically forced at frequency $\omega_{\mathrm{f}} \approx n \omega_{0}$ where $n$ is an integer.
The equation for $A$ admits then the universal form

$$
\begin{aligned}
A_{t}= & (\mu+i \nu) A+(1+i \alpha) A_{x x} \\
& -(1+i \beta)|A|^{2} A+\gamma_{n} A^{* n-1},
\end{aligned}
$$

where the subscripts $t$ and $x$ denote partial derivatives with respect to time and space, and all the parameters are real. The proximity to the Hopf bifurcation implies $\mu \ll 1$. The amplitude equation (3) can be derived for specific models using standard methods [20]. The general form of the equation can be deduced from symmetry considerations. In particular, the forcing term $A^{* n-1}$ follows from the discrete time translation symmetry, $t \rightarrow$ $t+2 \pi / \omega_{\mathrm{f}}$ (the amplitude equation should be invariant under the transformation $A \rightarrow A \exp i 2 \pi / n)$ [21].

In practical situations the periodic forcing often contains harmonics of the main frequency $\omega_{\mathrm{f}}$. Thus, forcing a system at $\omega_{\mathrm{f}} \approx \omega_{0}$, for example, may contain forcing components at $2 \omega_{0}, 3 \omega_{0}, \ldots$, and the corresponding terms, $\gamma_{2} A^{*}, \gamma_{3} A^{* 2}, \ldots$ in the amplitude equation should be considered as well. 


\section{THE $2: 1$ RESONANCE}

\section{A. The NIB Bifurcation}

An oscillating system that is forced at approximately twice its natural frequency is described by the amplitude equation (3) with $n=2$. This equation has two stable phase states (uniform solutions) whose phases $\arg (A)$ differ by $\pi$. In addition, the equation supports front solutions connecting the two phase states as the spatial coordinate goes from $-\infty$ to $+\infty$. The front solutions of Eq. (3) have been studied by Coullet et al. [12]. Consider first the gradient version of this equation, obtained by setting $\nu=\alpha=\beta=0[22]$

$$
A_{t}=\mu A+A_{x x}-|A|^{2} A+\gamma_{2} A^{*} .
$$

The term "gradient" refers to the existence of a Lyapunov (or free energy) functional for (4) which is minimized by the dynamics [23]. The two stable phase states of Eq. (4) are $A= \pm A_{0}$ where $A_{0}=$ $\sqrt{\mu+\gamma_{2}}$. One type of front solution connecting these states exists for all (positive) $\gamma_{2}$ values. It is given by

$$
A(x)=I(x ; \sigma)=\sigma A_{0} \tanh \left(\frac{1}{\sqrt{2}} A_{0} x\right)
$$

where $\sigma= \pm 1$ is the front polarity associated with the reflection symmetry $x \rightarrow-x$ of the Eqs. (3) and (4). The front solution (5) has a zero phase, $\arg (I)=0$, and is referred to as Ising front in analogy to Ising walls in ferromagnets.

At $\gamma_{2}=\mu / 3$ the Ising front loses stability and a pair of new fronts solutions appear (for a given polarity)

$$
A(x)=B_{ \pm}(x ; \sigma)=\sigma A_{0} \tanh (k x) \pm i \eta \operatorname{sech}(k x),
$$

where $\eta=\sqrt{\mu-3 \gamma_{2}}$ and $k=\sqrt{2 \gamma_{2}}$. The phases, $\arg \left(B_{ \pm}\right)$, associated with these front solutions are not zero but rather rotating clockwise and anticlockwise by $\pi$ as $x$ increases from $-\infty$ to $+\infty$.
It is in this sense that these front solutions resemble the Bloch domain walls in ferromagnets. They are consequently referred to as Bloch fronts.

Because of the gradient nature of (4) and the symmetry of the two phase states, all fronts solutions are stationary. The remarkable finding of Coullet et al. [12] was that any of the nongradient terms in Eq. (3) with $n=2$ whose coefficients are $\nu$, $\alpha$ and $\beta$ makes the two Bloch fronts propagating in opposite directions while leaving the Ising front stationary. The coexistence of counter-propagating Bloch fronts is a nongradient effect that does not exist in equilibrium systems. The Bloch front velocities are given by

$$
c= \pm \sigma \frac{3 \pi \eta A_{0}}{2 k\left(3 \mu-\gamma_{2}\right)}\left(-\nu+\beta \mu+(\alpha-\beta) \gamma_{2}\right) .
$$

Figure 2 shows a bifurcation diagram for the NIB bifurcation based on Eq. (7). Equation (7) can be derived by writing a front solution of Eq. (3) with $n=2$ in the form

$$
A(x, t)=A_{0}(x-c t)+\epsilon R,
$$

assuming the coefficients $\nu, \alpha$, and $\beta$ are of order $\epsilon \ll 1$. Here, $A_{0}(z)$ is either the Ising front solution

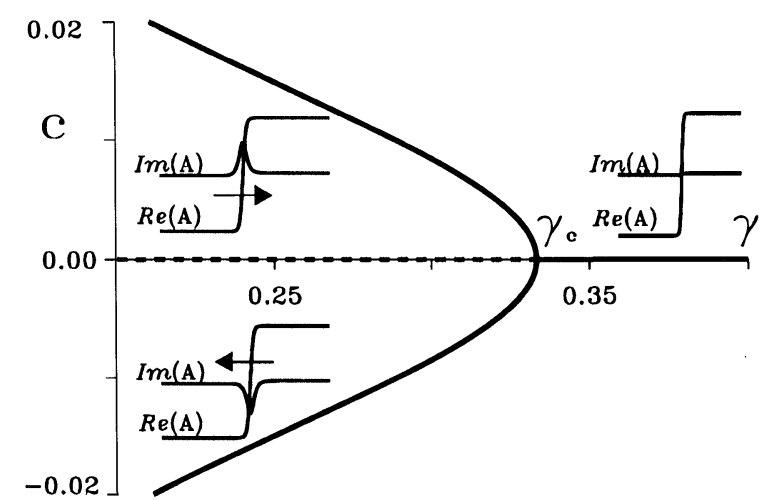

FIGURE 2 The NIB bifurcation for front solutions of Eq. (3) with $n=2$. For $\gamma>\gamma_{c}=\mu / 3$ there is a single stable Ising front with zero speed (solid line). For $\gamma<\gamma_{c}$ the Ising front is unstable (dashed line) and a pair of stable counterpropagating Bloch fronts appears (solid lines). Parameters: $\mu=1.0, \nu=0.01, \alpha=\beta=0.0$. (The parameter $\gamma$ is $\gamma_{2}$ in the text.) 

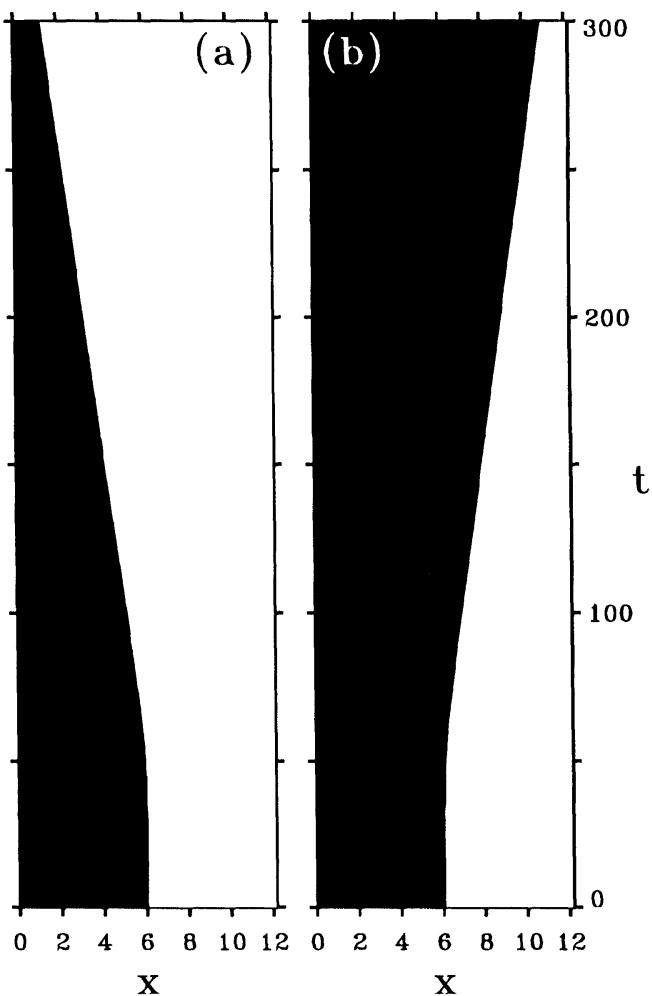

FIGURE 3 The NIB bifurcation in the 2:1 resonance: space-time plots of arg $(A)$ showing an unstable stationary Ising front evolving into left (a) and right (b) traveling Bloch fronts beyond the NIB bifurcation.

of (4), $I(z ; \sigma)$, or the Bloch front solutions, $B_{ \pm}(z ; \sigma)$, and $R$ represents higher order corrections. Using this form in (3) and applying solvability conditions at order $\epsilon$ lead to the result $c=0$ for $A_{0}=I$, and the expression (7) for $A_{0}=B_{ \pm}$. Another view of the NIB bifurcation is shown in Fig. 3 which shows space time plots of unstable Ising fronts that evolve into counter-propagating Bloch fronts. As will be discussed in the next section the NIB front bifurcation has significant effects on pattern formation.

\section{B. Implications on Pattern Formation}

Before embarking on pattern formation aspects of the amplitude equation (3) (with $n=2$ ) let us consider the more generic case where the two uniform phase states are not symmetric (under $A \rightarrow-A$ ).
The symmetry can be broken by adding to the forcing a component at the system's oscillation frequency, $\omega_{\mathrm{f}} \approx \omega$. The amplitude equation will now read

$$
\begin{aligned}
A_{t}= & (\mu+i \nu) A+(1+i \alpha) A_{x x} \\
& -(1+i \beta)|A|^{2} A+\gamma_{2} A^{*}+\gamma_{1} .
\end{aligned}
$$

The effect of the $1: 1$ forcing term, $\gamma_{1}$, is to grant velocities to all front solutions, even in the gradient case. The expression (7) for the Bloch front velocities now reads

$$
\begin{aligned}
c= & \pm \sigma \frac{3 \pi \eta A_{0}}{2 k\left(3 \mu-\gamma_{2}\right)}\left(-\nu+\beta \mu+(\alpha-\beta) \gamma_{2}\right) \\
& +\frac{3 \sigma A_{0} \gamma_{1}}{k\left(3 \mu-\gamma_{2}\right)},
\end{aligned}
$$

assuming $\gamma_{1}$ is small.

Imagine now a pair of Ising fronts with different polarities $\sigma$. Such a pair forms a domain of one phase state in a background of the other. Depending on the sign of $\gamma_{1}$ this domain either expands or shrinks. In both cases a uniform phase state will eventually prevail. In the symmetric case, $\gamma_{1}=0$, the attractive interactions between Ising fronts should lead in principle to a uniform state, but since the interactions are exponentially small most often patterns appear as frozen standing-wave patterns. The situation changes beyond the NIB bifurcation $\left(\gamma_{2}<\mu / 3\right)$ where counter-propagating Bloch fronts coexist. For now, a combination of two distinct Bloch fronts with different polarities form a traveling domain. Due to the different propagation speeds of the two Bloch fronts the traveling domain may either expand or shrink. In the former case a uniform state will be reached, but the shrinking domain may reach an equilibrium shape that travels invariably, because of the repulsive interactions between Bloch fronts. Asymptotic traveling domain solutions are shown in Fig. 4. Thus, the coexistence of the counter-propagating Bloch fronts allows for asymptotic traveling patterns [13].

The interaction between a pair of Bloch fronts and the formation of a stable traveling domain can 


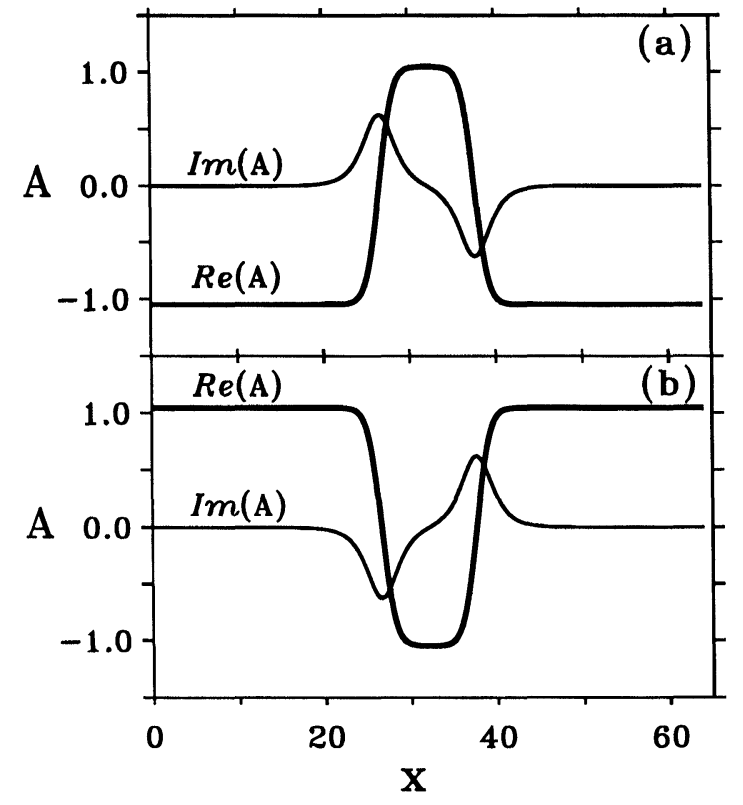

FIGURE 4 Traveling domain solutions to Eq. (9) near the NIB bifurcation. (a) A stable "up" domain, $\gamma_{1}<0$. (b) A stable "down" domain $\gamma_{1}>0$.

be studied by writing a traveling domain solution as [13]

$$
\begin{aligned}
A(x, t)= & B_{+}\left[x-x_{1}(t) ;+1\right]+B_{-}\left[x-x_{\mathrm{r}}(t) ;-1\right] \\
& -A_{0}+R(x, t),
\end{aligned}
$$

where the variables, $x_{\mathrm{r}}$ and $x_{1}$, are the positions of the leading (right) and trailing (left) Bloch fronts, $B_{ \pm}$are given by (6), and $R$ is a small correction term. The two polarities $(\sigma= \pm 1)$ are necessary to construct a domain bounded by the fronts. The two types of Bloch fronts, $B_{-}$and $B_{+}$, make the domain traveling. We assume that the system is nearly symmetric $\left(\left|\gamma_{1}\right| \ll 1\right)$, that it is close to the NIB bifurcation and that the domain is much wider than the width of the fronts. Following the methods of [24] the following equation for the domain width, $L=$ $x_{\mathrm{r}}-x_{1}$, has been derived:

$$
k A_{0} \dot{L}=3 \gamma_{1}-12 A_{0}^{3} \mathrm{e}^{-2 k L}+6 A_{0} \eta^{2} \mathrm{e}^{-k L} .
$$

The first term on the right hand side describes the effect of the broken symmetry between the two

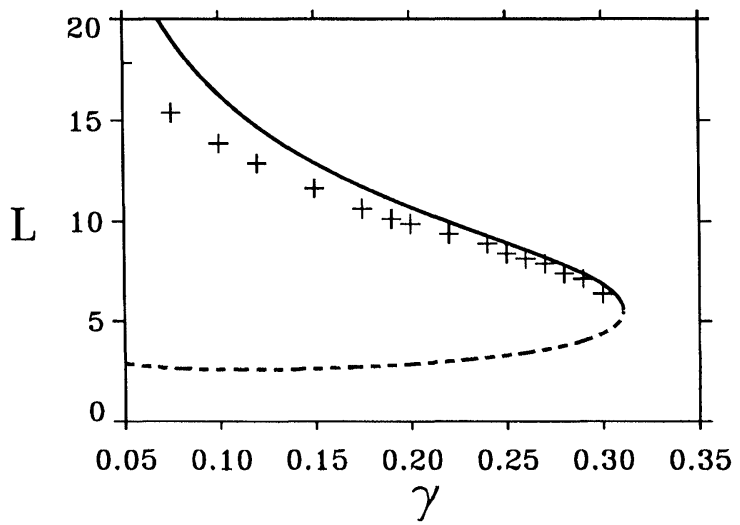

FIGURE 5 The distance, $L$, between the front and back of a traveling domain solution for $\gamma_{2}$ near the NIB bifurcation. The solid and dashed lines represent the stable and unstable branches solutions from Eq. (13). The crosses are data from direct numerical solution of Eq. (9). Parameters: $\mu=1, \nu=$ $0.01, \gamma_{1}=-0.001, \alpha=\beta=0$. (The parameter $\gamma$ is $\gamma_{2}$ in the text).

Bloch fronts; the initial domain expands $\left(\gamma_{1}>0\right)$ or shrinks $\left(\gamma_{1}<0\right)$ in time when the leading front is faster or slower than the trailing one. The second term describes an attractive front interaction generated by the real parts of the Bloch front solutions. The last term, generated by the imaginary parts of the Bloch front solutions, describes a longer range repulsive interaction. The repulsive interaction strengthens as $\gamma_{2}$ is decreased below the NIB bifurcation point, $\gamma_{2}=\mu / 3$, and becomes dominant at sufficiently small $\gamma_{2}$ values.

Solutions describing domains traveling at constant speeds are obtained by setting $\dot{L}=0$ in (12). The solutions to the resulting quadratic equation in $z=\exp (-k L)$ are

$$
L=-k^{-1} \ln \left(\eta^{2} \pm \sqrt{\eta^{4}+4 A_{0} \gamma_{1}}\right)+2 k^{-1} \ln 2 A_{0} .
$$

Consider the case where wide domains shrink, or $\gamma_{1}<0$. At the NIB bifurcation point, $\eta^{2}=$ $\mu-3 \gamma_{2}=0$, traveling domain solutions do not yet appear unless $\gamma_{1}=0$. They appear in a saddle-node bifurcation only for $\gamma_{2}<\gamma_{p}\left(\gamma_{1}\right) \leq \mu / 3$ where $\gamma_{p}\left(\gamma_{1}\right)$ solves $\left(\mu-3 \gamma_{p}\right)^{2}=4 \sqrt{\mu+\gamma_{p}}\left|\gamma_{1}\right|$. Graphs of these solutions in the $L-\gamma_{2}$ plane are shown in Fig. 5. The upper and lower branches represent stable and 
unstable solutions. Also shown in Fig. 5 are results from direct numerical solutions of Eq. (9) showing the stable traveling domain branch. The shape of the stable traveling domain is shown in Fig. 4(a).

In two space dimensions the NIB bifurcation has another interesting implication: it allows for spiral wave solutions. Figure 6 shows the time evolution

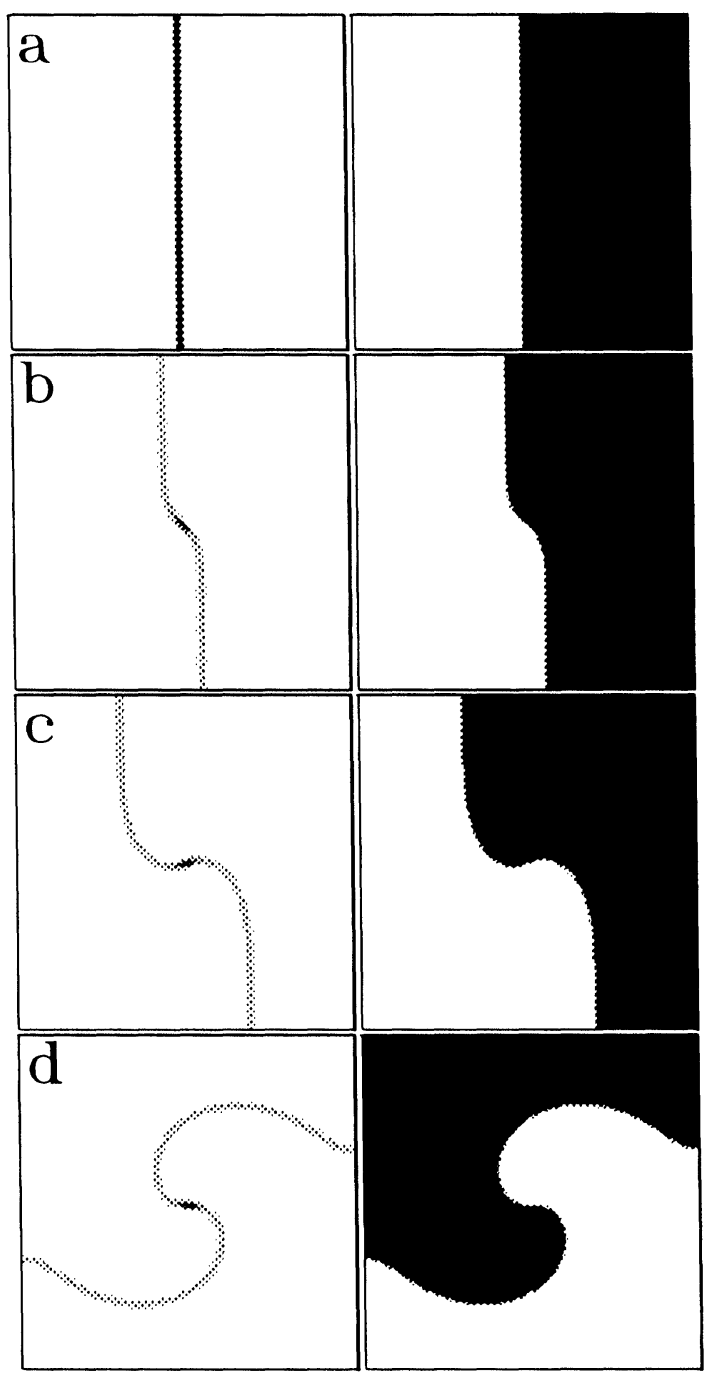

FIGURE 6 Simulation of the two-dimensional version of Eq. (3) with $n=2$ showing the development of a rotating spiral wave beyond the NIB bifurcation $\left(\gamma_{2}<\mu / 3\right)$. The left column is $|A|$ and the right column $\arg (A)$ in the $x-y$ plane. (a) An unstable Ising front consisting of two segments perturbed so as to initiate convergence to different Bloch fronts. (b), (c) and (d) The subsequent evolution toward a rotating spiral wave. of a $\pi$-front for $\gamma_{2}<\mu / 3$. The initial front consists of two segments that converge to different Bloch fronts. This leads to a twist motion that evolves into a rotating spiral wave [29].

\section{THE $4: 1$ RESONANCE}

\section{A. The Decomposition Instability}

The dynamics within the $4: 1$ resonance tongue is governed (close to the Hopf bifurcation and for small detuning) by Eq. (3) with $n=4$. The parameter $\mu$ can be scaled out by rescaling time space and amplitude as $t=\mu \tau, x=\sqrt{\mu / 2 z}$ and $B=A / \sqrt{\mu}$

$$
\begin{aligned}
B_{t}= & \left(1+i \nu_{0}\right) B+\frac{1}{2}(1+i \alpha) B_{x x} \\
& -(1+i \beta)|B|^{2} B+\gamma_{4} B^{* 3},
\end{aligned}
$$

where $\nu_{0}=\nu / \mu$. Consider first the gradient version obtained by setting $\nu_{0}=\alpha=\beta=0$ :

$$
B_{t}=B+\frac{1}{2} B_{x x}-|B|^{2} B+\gamma_{4} B^{* 3} .
$$

Equation (15) has four stable phase states for $0<\gamma_{4}<1$ shown by solid circles in Fig. 7: $B_{ \pm 1}= \pm \lambda$

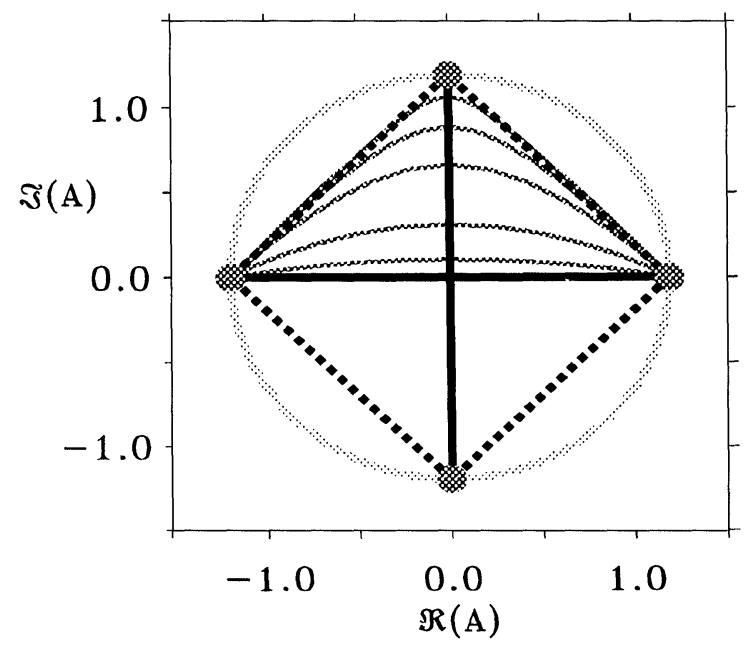

FIGURE 7 Uniform states and front solutions of Eq. (15) in the complex $B$ plane. The dots represent the 4 spatially uniform phase states. The solid lines are the $\pi$-front solutions and the dashed lines are the $\pi / 2$-fronts. The thin lines in the circle are phase portraits of front solutions at successive time steps showing the collapse of a $\pi$-front into a pair of $\pi / 2$-fronts. 
and $B_{ \pm i}= \pm i \lambda$, where $\lambda=1 / \sqrt{1-\gamma_{4}}$. Front solutions connecting pairs of these states divide into two groups, $\pi$-fronts and $\pi / 2$-fronts. The $\pi$-fronts, shown in Fig. 7 as solid lines, are given by

$$
\begin{aligned}
B_{-1 \rightarrow+1} & =B_{+1} \tanh x, \\
B_{-i \rightarrow+i} & =B_{+i} \tanh x .
\end{aligned}
$$

The $\pi / 2$-fronts are shown in Fig. 7 by the dashed curves. For the particular parameter value $\gamma_{4}=\frac{1}{3}$ they have the simple forms

$$
\begin{aligned}
& B_{+1 \rightarrow+i}=\frac{1}{2} \sqrt{\frac{3}{2}}[1+i-(1-i) \tanh x], \\
& B_{-i \rightarrow+1}=\frac{1}{2} \sqrt{\frac{3}{2}}[1-i+(1+i) \tanh x], \\
& B_{+i \rightarrow-1}=-B_{-i \rightarrow+1}, \\
& B_{-1 \rightarrow-i}=-B_{+1 \rightarrow+i} .
\end{aligned}
$$

Additional front solutions follow from the invariance of Eq. (15) under reflection, $x \rightarrow-x$. For example, the symmetric counterparts of $B_{+i \rightarrow+1}(x)$ and $B_{+1 \rightarrow-i}(x)$ are $B_{+1 \rightarrow+i}(x)=B_{+i \rightarrow+1}(-x)$ and $B_{-i \rightarrow+1}(x)=B_{+1 \rightarrow-i}(-x)$.

Consider now the nongradient system (14). The main effect of the nongradient terms is to make the $\pi / 2$-fronts traveling. The nongradient terms have no effect on the $\pi$-fronts which remain stationary. To see this assume a traveling solution $B(x-c t)$ of Eq. (14) and project this equation on the translational mode $B^{\prime}$. For $\pi$-fronts the resulting condition

$$
c\left\langle B_{0}^{\prime 2}\right\rangle=0 \quad B_{0}(z)=\lambda \tanh z
$$

implies $c=0$ (the brackets denote integration over the whole line). For $\pi / 2$-fronts with $\gamma_{4}=\frac{1}{3}$ the following expression is found:

$$
\begin{aligned}
|c| & =\frac{\lambda}{\left\langle B_{0}^{\prime 2}\right\rangle}\left[\left(\nu_{0}-\frac{1}{2} \lambda^{2} \beta\right)\left\langle B_{0}^{\prime}\right\rangle-\frac{1}{2} \beta\left\langle B_{0}^{2} B_{0}^{\prime}\right\rangle\right] \\
& =\frac{3}{2}\left(\nu_{0}-\beta\right),
\end{aligned}
$$

where $\lambda=\sqrt{3 / 2}$. A perturbation analysis around $\gamma_{4}=\frac{1}{3}$ shows that the expression (19) for the speed remains valid for small deviations of $\gamma_{4}$ from $\frac{1}{3}$.

The $\pi$-fronts (16) are similar to the Ising front in the $2: 1$ resonance and like the Ising front they lose stability as the forcing strength, $\gamma_{4}$, is decreased. Stability analysis of the $\pi$-fronts indicates that the instability occurs at $\gamma_{4}=\frac{1}{3}$. The nature of the instability, however, is quite different. It is a degenerate instability leading to asymptotic solutions that are not smooth continuations of the unstable stationary $\pi$-fronts, unlike the NIB pitchfork bifurcation. Figure 8 shows a space-time plot of $\arg (A)$ analogous to Fig. 3. The initial unstable $\pi$-front decomposes into a pair of $\pi / 2$-fronts traveling to the

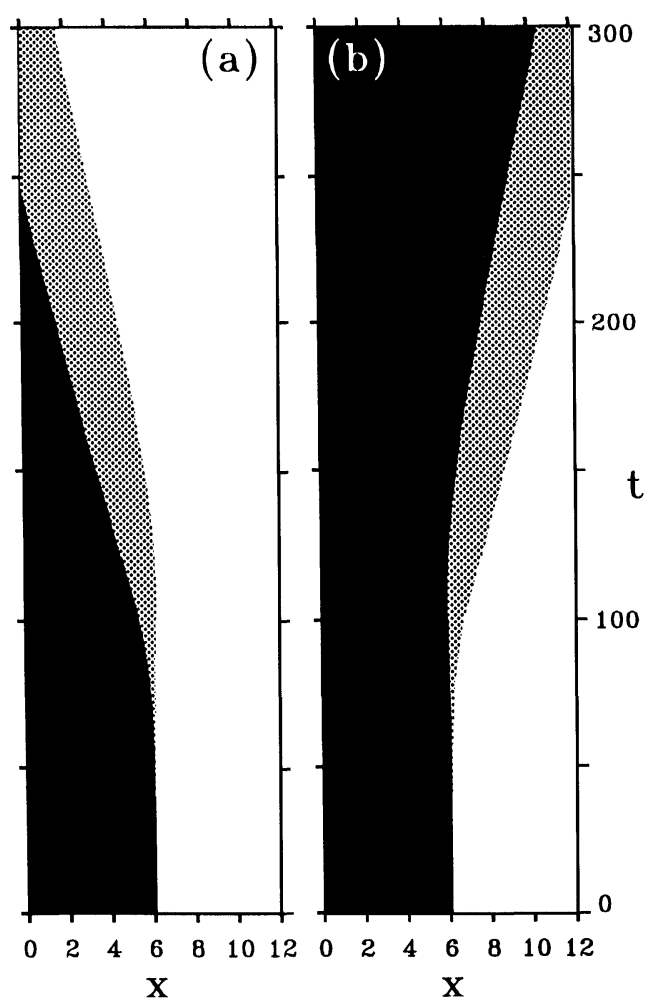

FIGURE 8 The decomposition instability in the $4: 1$ resonance: Space-time plots of $\arg (A)$ (solutions of Eq. (3) with $n=4$ ) showing the decomposition of an unstable $\pi$-front into a pair of $\pi / 2$-fronts traveling to the left (a) or to the right (b). The pairs of $\pi / 2$-fronts enclose grey colored domains whose oscillation phases are shifted by $\pi / 2$ with respect to the black and white domains. Parameters in Eq. (3): $\mu=1.0, \nu=0.02$, $\gamma_{4}=0.3$. 
right or to the left depending on initial conditions. Along with the $\pi$-front decomposition an intermediate phase state (the grey domain) appears. This behavior is found arbitrarily close to the instability point, and in this sense the new solutions are not smooth continuations of the $\pi$-front solution. The instability has been analyzed by Elphick et al. $[15,25]$ using Eq. (14) near $\gamma_{4}=\frac{1}{3}$. A brief description of this analysis follows.

Again, we consider first the gradient version (15). Introducing the new variables

$$
U=\Re(B)+\Im(B) \quad V=\Re(B)-\Im(B),
$$

Equation (15) is written as

$$
\begin{aligned}
& U_{t}=U+\frac{1}{2} U_{x x}-\frac{2}{3} U^{3}-\frac{d}{2}\left(U^{2}-3 V^{2}\right) U, \\
& V_{t}=V+\frac{1}{2} V_{x x}-\frac{2}{3} V^{3}-\frac{d}{2}\left(V^{2}-3 U^{2}\right) V
\end{aligned}
$$

where

$$
d=\gamma_{4}-1 / 3
$$

At the instability point, $\gamma_{4}=\frac{1}{3}$, the two equations decouple and admit solutions of the form

$$
\begin{aligned}
& U=\sigma_{1} B_{0}\left(x-x_{1}\right), \\
& V=\sigma_{2} B_{0}\left(x-x_{2}\right),
\end{aligned}
$$

where $B_{0}(x)=\sqrt{3 / 2} \tanh x, \sigma_{1,2}= \pm 1$, and $x_{1}$ and $x_{2}$ are arbitrary constants. An intuitive understanding of this family of solutions can be obtained by expressing these solutions back in terms of the complex amplitude $B$. For $\sigma_{1}=-\sigma_{2}=1$ for example, the solution (22) is equivalent to

$$
\begin{aligned}
B\left(x ; x_{1}, x_{2}\right)= & B_{-i \rightarrow+1}\left(x-x_{1}\right) \\
& +B_{+1 \rightarrow+i}\left(x-x_{2}\right)-\lambda .
\end{aligned}
$$

When $\left|x_{2}-x_{1}\right| \rightarrow \infty$ this form approaches a pair of isolated $\pi / 2$-fronts:

$$
B \approx B_{-i \rightarrow+1}\left(x-x_{1}\right), \quad x \approx x_{1},
$$

and

$$
B \approx B_{+1 \rightarrow+i}\left(x-x_{2}\right), \quad x \approx x_{2} .
$$

When $x_{2}-x_{1}=0$ it reduces to the $\pi$-front $B_{-i \rightarrow+i}$. Defining a mean position, $\zeta$, and an order parameter, $\chi$, by

$$
\zeta=\frac{1}{2}\left(x_{1}+x_{2}\right), \quad \chi=\frac{1}{2}\left(x_{2}-x_{1}\right),
$$

the one-parameter family of solutions, $\{\tilde{B}(x ; \zeta, \chi) \mid$ $\chi \in R\}$, where $\tilde{B}(x ; \zeta, \chi)=B\left(x ; x_{1}, x_{2}\right)$, represents $\pi / 2$-front pairs with distances, $2 \chi$, ranging from zero to infinity.

For $\left|\gamma_{4}-\frac{1}{3}\right|=|d| \ll 1$, the weak coupling between the two Eqs. (21a) and (21b) induces slow drift along the solution family $B\left(x ; x_{1}, x_{2}\right)$. A pair solution is now written as

$$
\begin{aligned}
& U=\sigma_{1} B_{0}\left[x-x_{1}(t)\right]+u, \\
& V=\sigma_{2} B_{0}\left[x-x_{2}(t)\right]+v,
\end{aligned}
$$

where $u$ and $v$ are corrections of order $d$. Equations of motion for $x_{1}$ and $x_{2}$ or for $\zeta$ and $\chi$ follow by inserting these forms in Eqs. (21a) and (21b) and applying solvability conditions at order $d$ :

$$
\begin{aligned}
& \dot{\zeta}=0, \\
& \dot{\chi}=-\frac{\mathrm{d} V}{\mathrm{~d} \chi}, \quad V=\frac{27}{16} d \int^{\chi} J(z) \mathrm{d} z,
\end{aligned}
$$

where

$$
\begin{aligned}
& J(\chi)=6\left(a^{-1}-a^{-3}\right)+\left(1-3 a^{-2}\right) G(a), \\
& G(a)=\left(1-a^{-2}\right) \ln \left(\frac{1+a}{1-a}\right)
\end{aligned}
$$

with $a=\tanh 2 \chi$. Note that Eqs. (24) and (25) are valid to all orders in $\chi$ and to linear order around $\gamma_{4}=\frac{1}{3}$. Figure 9 shows the potential $V(\chi)$ for $d>0$ $\left(\gamma_{4}>\frac{1}{3}\right)$ and $d<0$. There is only one extremum point, $\chi=0$, of $V$. For $d>0$ it is a minimum and $\chi$ converges to zero. Pairs of $\pi / 2$-fronts with arbitrary initial separation, $x_{2}-x_{1}$, attract one another and 


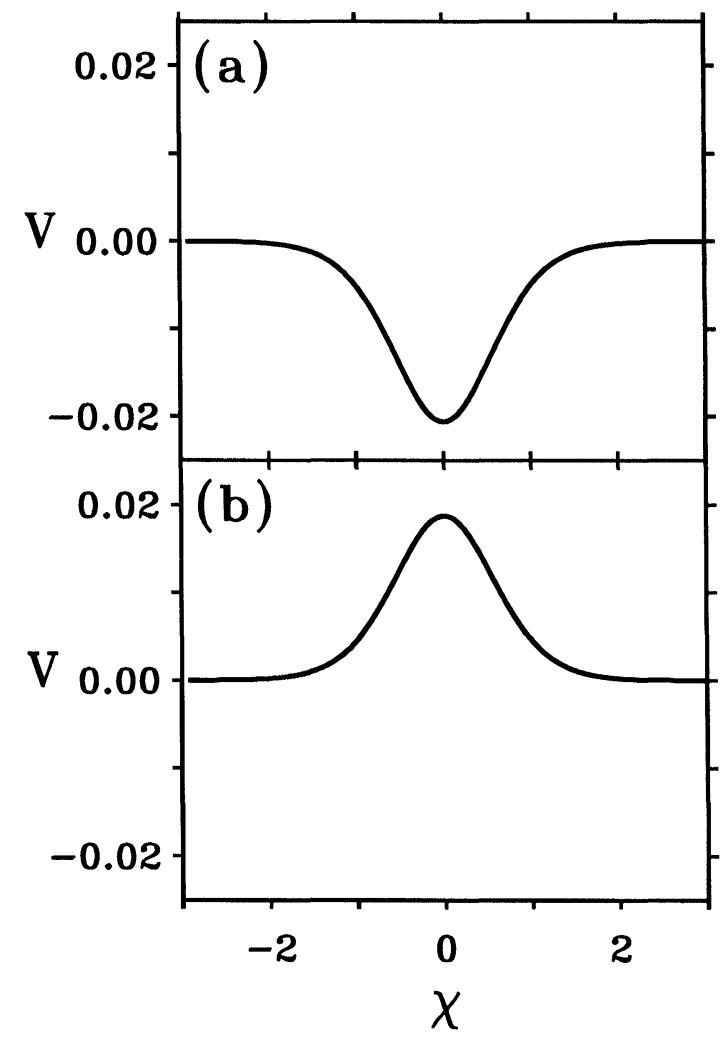

FIGURE 9 The potential $V(\chi)$. (a) For $d>0$ the extremum at $\chi=0$ is a minimum and $\chi$ converges to 0 (a $\pi$-front). (b) For $d<0$ the extremum is a maximum and $\chi$ diverges to $\pm \infty$ (isolated pair of $\pi / 2$-fronts).

eventually collapse to a single $\pi$-front $\left(x_{1}=x_{2}\right.$ or $\chi=0$ ). In practice, the collapse process is noticeable only for relatively small separations. For $d<0$ the extremum point, $\chi=0$, is a maximum and $\chi$ diverges to $\pm \infty$. A $\pi$-front decomposes into a pair of $\pi / 2$-fronts which repel one another as shown in Fig. 8 for the nongradient system (3). In the gradient case both $\pi$ and $\pi / 2$-fronts are stationary (in the absence of interactions). Since the potential $V(\chi)$ becomes practically flat at finite $\chi$ values, the pair of $\pi / 2$-fronts do not seem to depart from one another at long times. Figure 7 shows the decomposition process of a $\pi$-front in the complex $B$ plane. Starting with the $B_{-1 \rightarrow+1} \pi$-front, represented by the thick solid phase portrait, the time evolution (thin solid phase portraits) is toward the fixed point $B_{+i}$ and the dashed phase portraits representing the pair of $\pi / 2$-fronts $B_{+1 \rightarrow+i}$ and $B_{+i \rightarrow-1}$. Because of the parity symmetry $\chi \rightarrow-\chi$, an appropriate perturbation of the initial $B_{-1 \rightarrow+1} \pi$-front could have led the dynamics toward the pair $B_{+1 \rightarrow-i}$ and $B_{-i \rightarrow-1}$. Notice that for $d=0, \dot{\zeta}=0, \dot{\chi}=0$, and we recover the two-parameter family of pair solutions $B(x ; \zeta, \chi)$ with arbitrary $\zeta$ and $\chi$.

The derivation of Eqs. (24) and (25) can easily be extended to the nongradient case assuming $\nu_{0}$, $\alpha$ and $\beta$ are small. The $\chi$ equation remains unchanged. The $\zeta$ equation takes the form

$$
\frac{\sigma_{2}}{\sigma_{1}} \dot{\zeta}=\nu F_{\nu}(\chi)+\alpha F_{\alpha}(\chi)+\beta F_{\beta}(\chi),
$$

where $F_{\nu}, F_{\alpha}$ and $F_{\beta}$ are odd functions of $\chi$ and do not vanish when $d=0$ [25]. When $|\chi| \rightarrow \infty$ the right hand side of (26) converges to $\frac{3}{2}\left(\nu_{0}-\beta\right)$, the speed of a $\pi / 2$-front solution of Eq. (14). The $\chi=0$ solution (representing a $\pi$-front) remains stationary $(\dot{\zeta}=0)$ in the nongradient case as well. At $\gamma_{4}=\frac{1}{3}$ $(d=0)$ it loses stability and decomposes into a pair of $\pi / 2$-fronts which approach the asymptotic speed $\frac{3}{2}\left(\nu_{0}-\beta\right)$.

The degeneracy of solutions at $\gamma_{4}=\frac{1}{3}$ is lifted by adding higher order terms to the amplitude equation (14). These terms are smaller by a factor of $\mu \ll 1$ than the terms appearing in (14) and their effect is noticeable only in a $\mu$-neighborhood of $\gamma_{4}=\frac{1}{3}$. Apart from this small parameter range the overall behavior does not change [25].

\section{B. Implications on Pattern Formation}

Like in the $2: 1$ resonance the $\pi$-front instability designates a transition from frozen standing waves to traveling waves. The coexistence of $\pi$-fronts and $\pi / 2$-fronts, however, allows in principle both twophase and four-phase patterns. The $\pi$-front decomposition instability contains information not only about the transition from standing to traveling waves, but also about the parameter regimes where two-phase and four-phase patterns are expected to be seen. For $\gamma_{4}>\frac{1}{3}$ the interaction between a pair of propagating $\pi / 2$-fronts is attractive as indicated 
by the single minimum of the potential $V(\chi)$ (see Fig. 9). The minimum at $\chi=0$ corresponds to a $\pi$-front and according to Eq. (26) the $\pi$-front is stationary. As a result, traveling four-phase patterns consisting of $\pi / 2$-fronts converge to stationary two-phase patterns consisting of $\pi$-fronts. When $\gamma_{4}<\frac{1}{3}$ the interaction between $\pi / 2$-fronts becomes repulsive. Stationary $\pi$-fronts, corresponding to the maximum of $V(\chi)$ at $\chi=0$, are unstable and decompose into pairs of traveling $\pi / 2$-fronts. As a result stationary two-phase patterns destabilize and evolve into four-phase traveling patterns.

Figure 10(a) shows a stably rotating four-phase spiral wave for $\gamma_{4}<\frac{1}{3}$. Figure $10(b-d)$ show the collapse of this spiral wave into a stationary twophase pattern as $\gamma_{4}$ is increased past $\frac{1}{3}$. The collapse begins at the spiral core where the $\pi / 2$-front interactions are the strongest. As pairs of $\pi / 2$-fronts attract and collapse into $\pi$-fronts, the core splits into two vertices that propagate away from each other leaving behind a two-phase pattern.

\section{HIGHER $2 n: 1$ RESONANCES}

Numerical studies of amplitude equations for higher resonances suggest the existence of $\pi$-front decomposition instabilities in $2 n: 1$ resonances with $n>1$. The following generalization has been conjectured: within the $2 n: 1$ tongue $(n>1)$, upon decreasing $\gamma_{2 n}$, a $\pi$-front may lose stability by decomposing into $n$ propagating $\pi / n$-fronts. Consider for example the equation

$$
\begin{aligned}
B_{t}= & \frac{1}{2} B_{x x}+\left(1+i \nu_{0}\right) B+\mu_{4}|B|^{2} B \\
& +\mu_{6}|B|^{4} B+\gamma_{4} B^{* 3}+\gamma_{6} B^{* 5} .
\end{aligned}
$$

The normal form equation up to fifth order contains many more terms whose coefficients were set to zero for simplicity. Figure 11 shows the decomposition in the complex $B$ plane of a $\pi$-front within the $6: 1$ tongue $\left(\gamma_{6} \neq 0\right)$ into three $\pi / 3$-fronts. Figure 12 shows a space-time plot of the decomposition instability within the $6: 1$ tongue. The initial unstable $\pi$-front decomposes into three

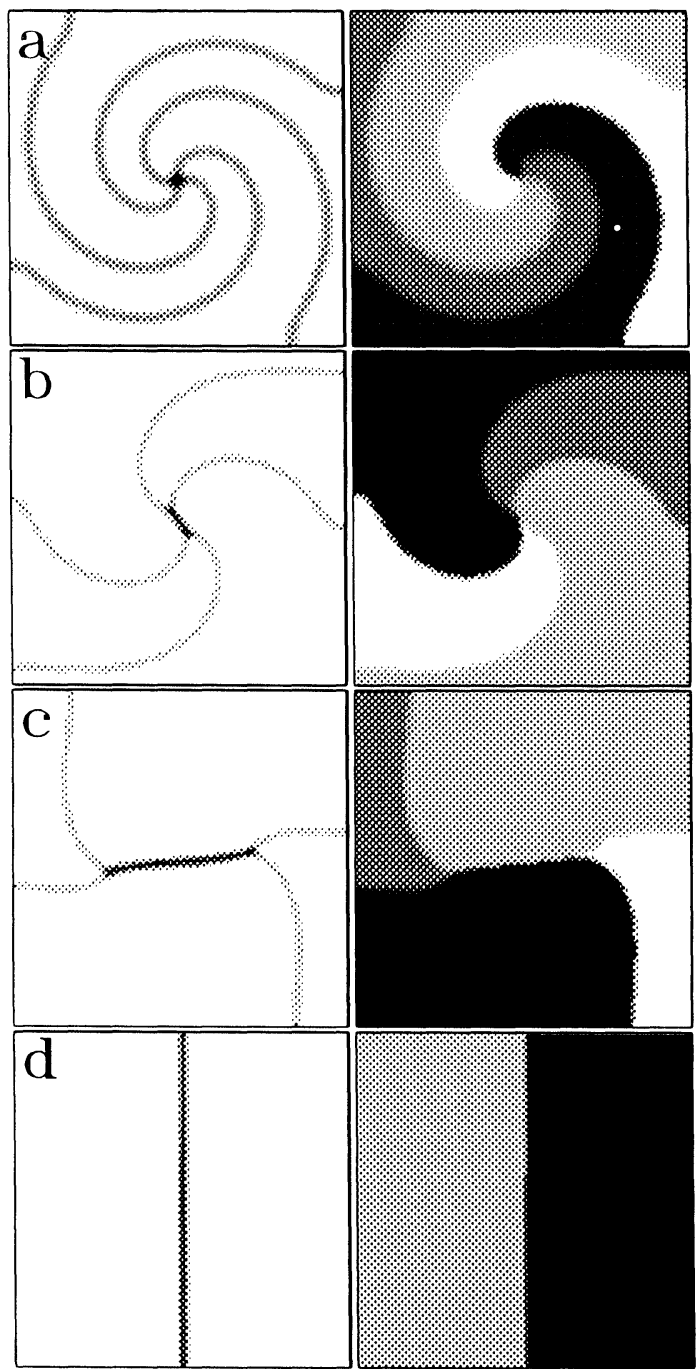

FIGURE 10 Numerical solution of a two-dimensional version of Eq. (14) showing the collapse of a rotating four-phase spiral-wave into a stationary two-phase pattern. The left column is $|A|$ and the right column $\arg (A)$ in the $x-y$ plane. (a) The initial four-phase spiral wave (computed with $\gamma_{4}<\frac{1}{3}$ ). (b) The spiral core, a 4-point vertex, splits into two 3-point vertices connected by a $\pi$-front. (c) A two-phase pattern develops as the 3-point vertices further separate. (d) The final stationary two-phase pattern. Parameters: $\gamma_{4}=0.6, \nu_{0}=0.1$, $\alpha=\beta=0, x=[0,64], y=[0,64]$.

$\pi / 3$-fronts, traveling to the left or to the right depending on initial conditions. Along with this process two intermediate phase states appear between the original white and black phases. A similar decomposition instability has been observed within the $8: 1$ tongue. 


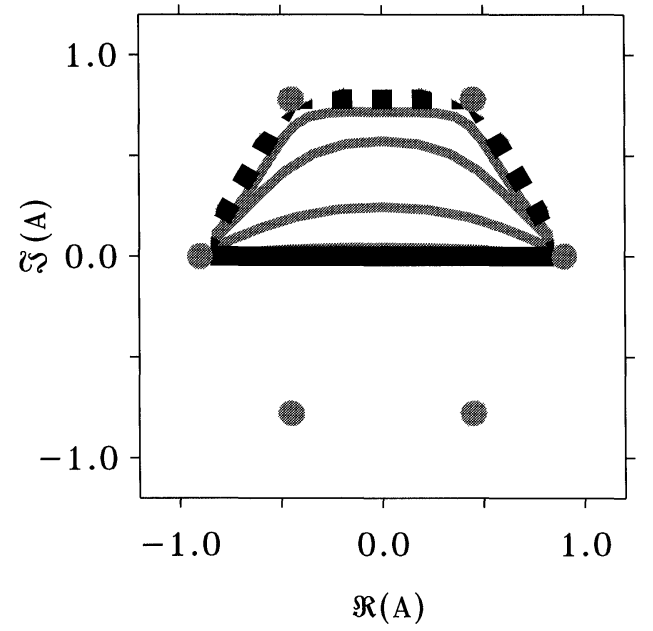

FIGURE 11 Phase portraits of front solutions at successive time steps (thin solid lines) showing the decomposition of a $\pi$-front (thick solid line) into three $\pi / 3$-fronts (dashed lines) in the $6: 1$ resonance tongue. Parameters in Eq. (27): $\gamma_{6}=0.9$, $\mu_{4}=-1.0, \mu_{6}=-1.0$. All other parameters are zero.
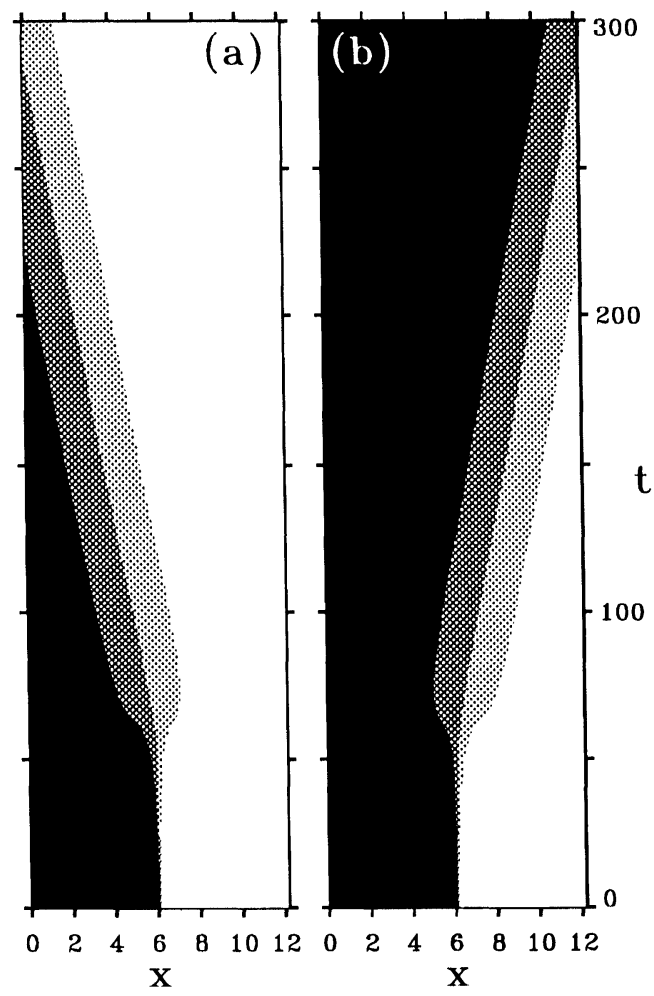

FIGURE 12 Decomposition of a $\pi$-front into three $\pi / 3$ fronts in the $6: 1$ resonance band. The figures show spacetime plots of $\arg (B)$ using numerical solutions of Eq. (27) with parameters $\gamma_{6}=0.9, \mu_{4}=-1.0, \mu_{6}=-1.0, \nu=0.1$. All other parameters are zero.

\section{THEORY VS. EXPERIMENT}

The main difficulty in confronting the available theory with the experiments on the forced Belousov-Zhabotinsky reaction is that the experiments were carried out far from the Hopf bifurcation while the theory is valid only close to the bifurcation. At high forcing strengths, within the 2:1 tongue, standing two-phase patterns were observed. This observation is consistent with the behavior in the Ising regime that the theory predicts. At sufficiently lower forcing strengths a transition to traveling waves is observed [16], as the theory predicts too. However, no indications for Bloch phase fronts and phased locked domains have so far been found. This behavior may be attributed to the relaxational nature of the oscillations far from the Hopf bifurcation, and consequently to large phase gradients that develop. These gradients may prevent convergence to the two uniform phase states at low forcing strengths.

Direct studies of the NIB bifurcation have been carried out on liquid crystals subjected to rotating magnetic fields $[17,18]$. This system, like forced oscillatory media, can be modeled by Eq. (3) with $n=2$ [17]. Experimental observations of decomposition instabilties of $\pi$-fronts have not been reported so far. The lowest resonance to display such an instability, $4: 1$, was beyond the scope of the experiments on the forced Belousov-Zhabotinsky reaction reported in [19].

Some of the experimental observations shown in Fig. 1 can nevertheless be accounted for using the available theory. The absence of multiple stable phase states within the 1:1 resonance does not allow for domain patterns as in higher resonances (although nonuniform phase dynamics arising from phase instabilities of the uniform state may occur). This is consistent with the uniform oscillations shown in the first pair of frames on the left. The two phase states that coexist within the $2: 1$ resonance allow for two-phase patterns as shown in the third and fourth pairs of frames (from left). These patterns are standing waves suggesting that these observations were taken at high illumination 
intensities within the Ising regime. The two other patterns shown in Fig. 1 (second and fifth pairs of frames) correspond to the $3: 2$ and $3: 1$ resonances. In both cases three uniform phase states coexist. Three phase patterns have been observed within the $3: 1$ resonance only. They consist of successive domains with shifts of $2 \pi / 3$ in the oscillation phases. The boundaries of these domains ( $2 \pi / 3$-fronts) were found to drift very slowly. In other experiments within the $3: 1$ tongue traveling three phase patterns were observed [16]. Numerical studies of Eq. (3) with $n=3$ have indeed found three phase traveling waves (spiral waves) [14].

Some other experimental observations are not yet understood. The bubble patterns within the $3: 2$ resonance (second pair of frames in Fig. 1) are not simple three-phase patterns although the power spectrum at any spatial point shows well defined peaks at multiples of $\omega_{\mathrm{f}} / 3$. The bubbles randomly appear and disappear and the mechanism that governs this behavior is not known. The transition to labyrinthine patterns within the $2: 1$ resonance as the forcing frequency, $\omega_{\mathrm{f}}$, is increased is also not fully understood. Simulations of a forced Brusselator model within the $2: 1$ resonance reproduced the transition to labyrinthine patterns [33,34]. The transition has been attributed to a transverse instability of an Ising front. Transverse front instabilities leading to labyrinthine patterns through fingering and tip spliting have been found earlier in bistable (unforced) reaction-diffusion models [26-28]. A neccessary condition is repulsive front interactions that rule out merging of growing fingers, but the origin of such interactions in the context of Eq. (3) is not clear. The decoration that appears on the experimental $2 \pi / 3$-fronts within the $3: 1$ resonance suggest the existence of a transverse instability but no theoretical account has yet been offered.

There are also theoretical predictions that have not been tested yet in experiments like the $\pi$-front decomposition instability in the $4: 1$ resonance and its implications on pattern formation. In the vicinity of the NIB bifurcation complex spatio-temporal behavior involving spontaneous nucleation of spiral waves may arise [27,29]. A theoretical account of this behavior has been given in the context of activator-inhibitor systems [30-32]. Complex spatio-temporal dynamics near the transition from standing to traveling waves within the $2: 1$ resonance have also been found experimentally in the periodically illuminated Belousov-Zhabotinsky reaction [16], but no attempt has yet been made to interpret these observations.

\section{CONCLUSION}

I have presented here a short review of recent studies of extended dissipative oscillatory media subjected to temporal periodic forcing. Most attention has been given to the analysis of $\pi$-front instabilities within even $2 n: 1$ resonances and to the implications they bear on pattern formation. Despite the theoretical progress described in this review many aspects of periodically forced oscillatory systems are still not understood. The discussion of experimental findings in the previous section points toward a few of them. In particular, the effect of the distance to the Hopf bifurcation has to be studied. Additional open questions include: (i) dynamics within overlapping resonances which becomes relevant at high forcing strengths, (ii) forcing at multiple frequencies, e.g. periodic forcing that contains harmonics and (iii) coupled front-phase dynamics (involving instabilities of uniform phase states) which may shed light on the onset of spatiotemporal chaotic behaviors.

I did not discuss here periodic forcing of nonoscillatory extended media such as excitable media [35] and granular systems [36-39]. Nor did I discuss conservative or nearly conservative systems that are periodically forced [40-44]. All these systems share common features but also differ in many aspects.

\section{References}

[1] A.T. Winfree (1980). The Geometry of Bilogical Time (Springer, New York).

[2] A.T. Winfree (1987). The Timing of Biological Clocks (Scientific American Books Inc., New York).

[3] R.J. Field and M. Burger (1985). Oscillations and Traveling Waves in Chemical Systems (Wiley, New York). 
[4] L. Glass and M.C. Mackey (1988). From Clocks to Chaos (Princeton University Press, Princeton).

[5] A. Goldbeter (1996). Biochemical Oscillations and Cellular Rhythms (Cambridge University Press, Cambridge).

[6] V.I. Arnold (1983). Geometrical Methods in the Theory of Ordinary Differential Equations (Springer, New York).

[7] M.J. Feigenbaum, L.P. Kadanoff and S.J. Shenker (1982). Physica D 5, 370 .

[8] D. Rand, S. Ostlund, J. Sethna and E. Siggia (1984). Physica D 6, 303

[9] M.H. Jensen, P. Bak and T. Bohr (1984). Phys. Rev. A 30, 1960.

[10] L. Glass and J. Sun (1994). Phys. Rev. E 50, 5077.

[11] D. Walgraef (1997). Spatio-Temporal Pattern Formation (Springer-Verlag, New York).

[12] P. Coullet, J. Lega, B. Houchmanzadeh and J. Lajzerowicz (1990). Phys. Rev. Lett. 65, 1352.

[13] C. Elphick, A. Hagberg, E. Meron and B. Malomed (1997). Phys. Lett. A 230, 33

[14] P. Coullet and K. Emilsson (1992). Physica D 61, 119.

[15] C. Elphick, A. Hagberg and E. Meron (1998). Phys. Rev. Lett. 80, 5007.

[16] A. Lin and H.L. Swinney, private communication.

[17] T. Frisch, S. Rica, P. Coullet and J.M. Gilli (1994). Phys. Rev. Lett. 72, 1471.

[18] S. Nasuno, N. Yoshimo and S. Kai (1995). Phys. Rev. E 51, 1598; K.B. Migler and R.B. Meyer (1994). Physica D 71, 412.

[19] V. Petrov, Q. Ouyang and H.L. Swinney (1997). Nature 388, 655 .

[20] M.C. Cross and P.C. Hohenberg (1993). Rev. of Mod. Phys. 65, 851 .

[21] C. Elphick, G. Iooss and E. Tirapegui (1987). Phys. Lett. A 120, 459.

[22] B.A. Malomed and A.A. Nepomnyashcy (1994). Europhys. Lett. 27, 649.

[23] P.C. Fife, "Pattern formation in gradient systems". In Handbook for Dynamical Systems. III: Toward Applications (B. Fiedler and N. Kopell, Eds.) Elsevier (to appear).
[24] C. Elphick, E. Meron and E.A. Spiegel (1990). SIAM J. Appl. Math. 50, 490.

[25] C. Elphick, A. Hagberg and E. Meron (1999). Phys. Rev. E. (to appear)

[26] T. Ohta, M. Mimura and R. Kobayashi (1989). Physica D 34, 115.

[27] A. Hagberg and E. Meron (1994). Chaos 4, 477.

[28] R.E. Goldstein, D.J. Muraki and D.M. Petrich (1996). Phys. Rev. E 53, 3933.

[29] T. Frisch and J.M. Gilli (1995). J. Phys. II France 5, 561.

[30] A. Hagberg and E. Meron (1996). Nonlinear Science Today, http://www.springerny.com:80/nst/nstarticles.html.

[31] A. Hagberg and E. Meron (1997). Phys. Rev. Lett. 78, 1166.

[32] A. Hagberg and E. Meron (1998). Physica D 123, 460.

[33] V. Petrov, M. Gustafsson and H.L. Swinney (1998). Formation of labyrinthine patterns in a periodically forced reaction-diffusion model, Proceedings of the Fourth Experimental Chaos Conference (World Scientific, Singapore) (to appear).

[34] A. Lin, V. Petrov, H.L. Swinney, A. Ardelea and G.F. Carey (1999). Resonant pattern formation in a spatially extended chemical system (preprint).

[35] M. Xie (1998). Theoretical results of forced excitable systems. Ph.D. Thesis, The University of Utah.

[36] P.B. Umbanhowar, F. Melo and H.L. Swinney (1996). Nature 382, 793.

[37] L.S. Tsimring and I.S. Aranson (1997). Phys. Rev. Lett. 79, 213.

[38] W. Losert, D.G.W. Cooper and J.P. Gollub. Propagating front in an excited granular layer. Phys. Rev. E. (submitted).

[39] J. Eggers and H. Riecke. A continuum description of vibrated sand (preprint).

[40] A. Kudrolli and J.P. Gollub (1996). Physica D 97, 133.

[41] W. Zhang and J. Viñals (1996). Phys. Rev. E 53, R4286.

[42] D. Binks and W. van de Water (1997). Phys. Rev. Lett. 78, 4043.

[43] P. Coullet, T. Frisch and G. Sonnino (1994). Phys. Rev. E 49, 2087.

[44] C. Szwaj, S. Bielawski, D. Derozier and T. Erneux (1998). Phys. Rev. Lett. 80, 3986. 


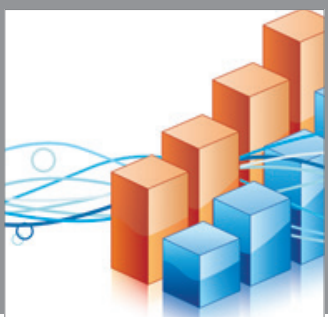

Advances in

Operations Research

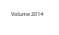

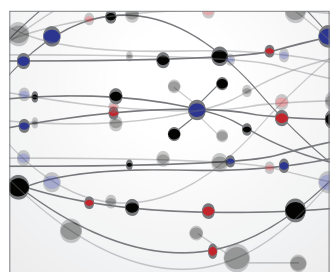

\section{The Scientific} World Journal
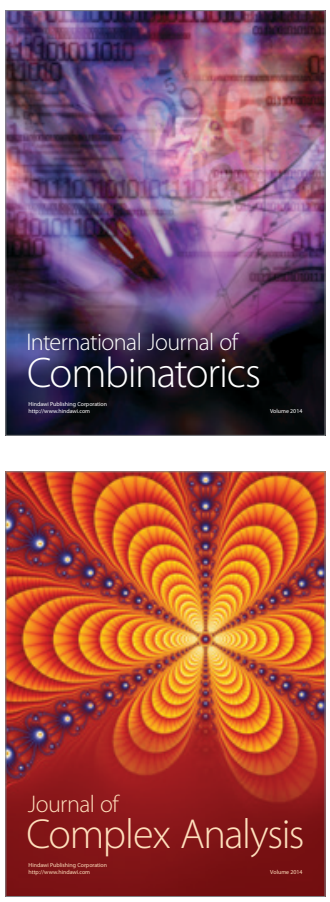

International Journal of

Mathematics and

Mathematical

Sciences
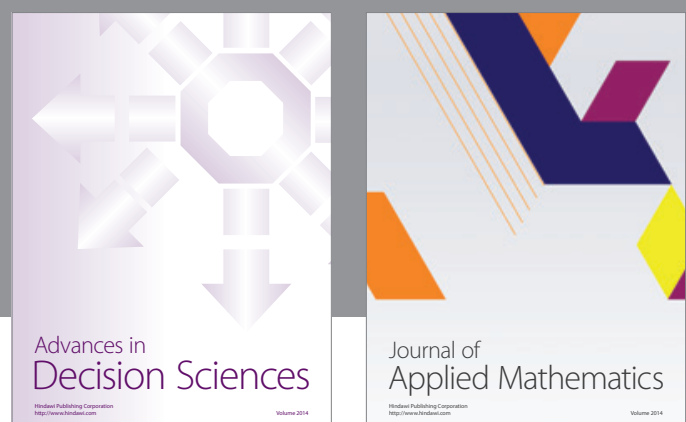

Journal of

Applied Mathematics
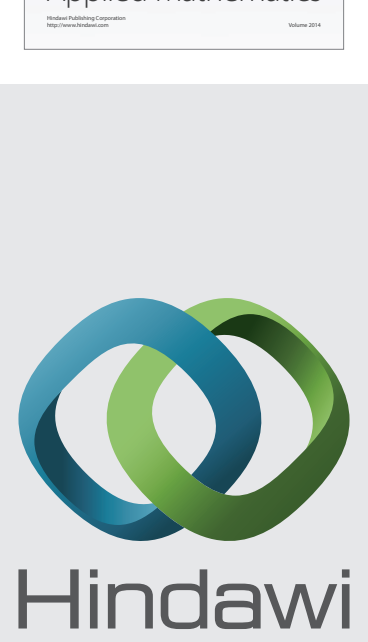

Submit your manuscripts at http://www.hindawi.com
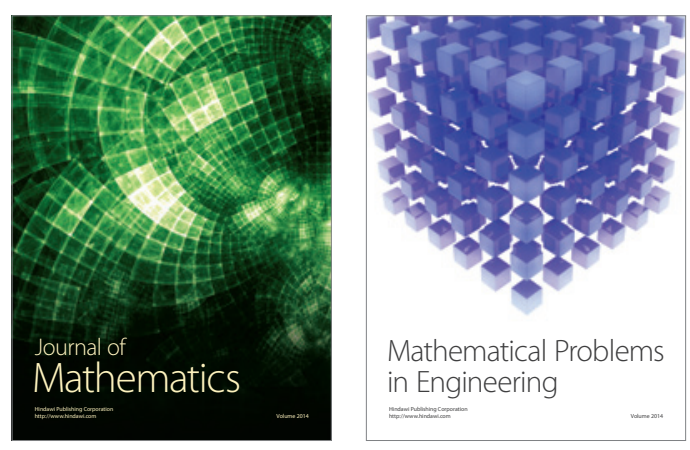

Mathematical Problems in Engineering
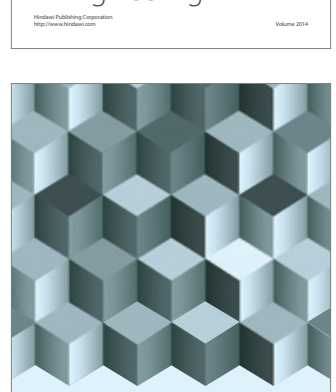

Journal of

Function Spaces
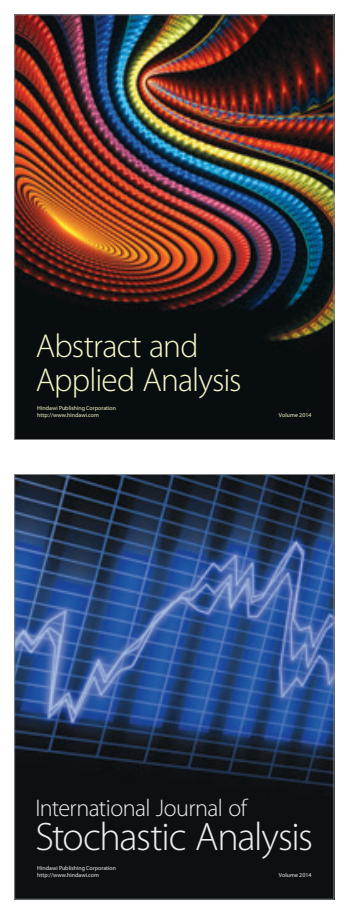

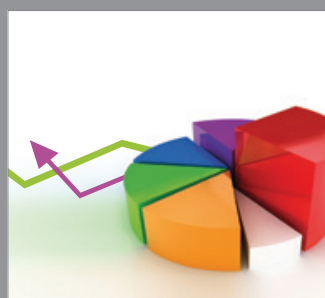

ournal of

Probability and Statistics

Promensencen
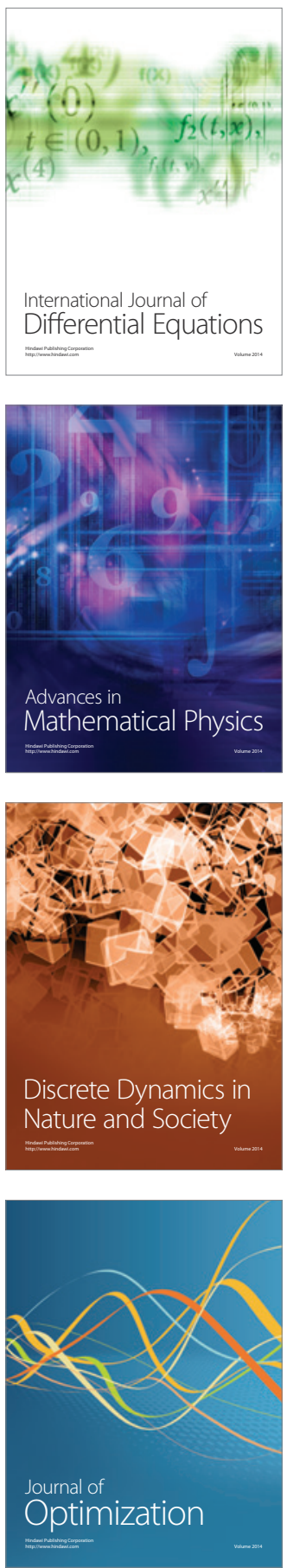